\title{
Clinical therapy of hyaluronic acid combined with platelet-rich plasma for the treatment of knee osteoarthritis
}

\author{
WENXING YU, PENG XU, GUILING HUANG and LIN LIU \\ Department of Orthopedics, Hong-Hui Hospital, Xi'an Jiangtong University College of Medicine, \\ Xi'an, Shaanxi 350021, P.R. China
}

Received December 19, 2016; Accepted August 23, 2017

DOI: 10.3892/etm.2018.6412

\begin{abstract}
Knee osteoarthritis is the most common degenerative disease of the joints caused by articular cartilage injury, degradation of the joint edge and subchondral bone hyperplasia. Various treatments are used to alleviate the symptoms of patients with knee osteoarthritis, including analgesics and intra-articular injections. Platelet-rich plasma (PRP) is an autologous and multifunctional platelet concentrate of the blood, which stimulates the cartilage healing process and improves the damage caused by articular disease. Hyaluronic acid (HA) is an effective treatment for patients with knee osteoarthritis. In the current study, the effectiveness of PRP and HA combination therapy administered via intra-articular injections for patients with knee osteoarthritis was analyzed. A total of 360 patients with knee osteoarthritis were randomized into four different treatment groups as follows: Double-blind treatment with PRP (2-14 ml); double-blind treatment with HA (0.1-0.3 mg); combination therapy of PRP and HA; and placebo groups. Following treatment, all patients were evaluated using the Western Ontario and McMaster Universities Arthritis Index (WOMAC) and Common Toxicity Criteria. The most common treatment-emergent adverse events were hypertension and proteinuria. The current study demonstrated that PRP and HA treatment significantly improved arthralgia, and PRP treatment was determined to be significantly more effective than HA treatment using the WOMAC pain score $(\mathrm{P}<0.05)$. PRP and HA combination treatment significantly improved arthralgia, reduced humoral and cellular immune responses and promoted angiogenesis, which improved the patients' histological parameters compared with PRP or HA treatment alone. These results suggested that PRP and HA combination treatment may be a potential treatment option for patients with knee osteoarthritis in the future.
\end{abstract}

Correspondence to: Professor Peng $\mathrm{Xu}$, Department of Orthopedics, Hong-Hui Hospital, Xi'an Jiangtong University College of Medicine, 46 Cangyi Road, Xi'an, Shaanxi 350021, P.R. China E-mail: xupengmedicine@163.com

Key words: platelet-rich plasma, hyaluronic acid, keen osteoarthritis, arthralgia

\section{Introduction}

Osteoarthritis is a degenerative disease with clinical manifestations, including joint pain, tenderness, stiffness, joint swelling, restricted movement and joint deformities (1). In recent years, the incidence of osteoarthritis has increased and presents as a serious threat to human health and quality of life (2-4). The causes of osteoarthritis are complex, therefore it is difficult to develop a comprehensive classification system and its pathogenesis remains unclear (5). Osteoarthritis is divided into primary and secondary osteoarthritis depending on the presence of local and systemic risk factors, including high bone mass and metabolic disorders (6). It is frequently diagnosed in the clinic as rheumatoid arthritis and ankylosing spondylitis (7). Previous studies have demonstrated that rheumatoid arthritis is the most common manifestation of osteoarthritis in patients; however, an effective treatment strategy for rheumatoid arthritis remains unknown $(8,9)$. Thus, further investigations into efficient treatments for osteoarthritis with minimal side effects are required.

Platelet-rich plasma (PRP) is a multifunctional platelet concentrate of the blood that may be used for the treatment of the manifestations of osteoarthritis, including osteonecrosis of the femoral head, cartilage injury and rheumatoid arthritis (10). It has been demonstrated previously that PRP improves the repair of articular cartilage injury in patients with joint disease by removing harmful inflammation factors (11). It was also demonstrated that PRP reduces the level of inflammatory factor synovial fluid in rheumatoid arthritis without exhibiting side effects (12). Treatment-emergent adverse events of PRP have not been previously reported. The isolation of PRP, including blood product rich in cytokines, growth factors and other bio-active molecules from autologous peripheral blood mononuclear cells, is an efficient and innovative treatment strategy (13). Furthermore, Sadabad et al (14) investigated the efficiency of PRP vs. hyaluronic acid (HA) for the treatment of knee osteoarthritis and Khoshbin et al (15) conducted a systematic review of PRP as a therapeutic intervention in the management of symptomatic knee osteoarthritis. These reports demonstrated that intravenous injection of PRP repaired the damage to the tendon and articular bone and reduced inflammation, which may serve a key function in maintaining the morphology, collagen microarchitecture and mechanical properties of the injected vein. 
$\mathrm{HA}$ is a high molecular weight glucosamine $\left(5-7 \times 10^{6} \mathrm{kD}\right)$ synthesized by chondrocytes, fibroblasts and synoviocytes (16). It is responsible for viscoelasticity and lubricance in the synovial fluid and extracellular matrix (17). It has been demonstrated that there is a higher concentration of HA $(2.5-4.0 \mathrm{mg} / \mathrm{ml})$ in the synovial fluid, while there is a decreased concentration level in patients with osteoarthritis (18). Therefore, the concentration of HA is an indicator of the prognosis of patients with osteoarthritis. The efficacy of HA treatment in improving osteoarthritis symptoms has been widely studied and the clinical outcomes for patients with osteoarthritis are positive (19-21).

The current study investigated the efficacy and outcomes of PRP and HA combination treatment in patients with knee osteoarthritis aged 22-68 years. The clinical data demonstrated that intra-articular injections of PRP were more successful in recovering articular function, alleviating symptoms and reducing arthralgia and body pain compared with HA treatment. PRP and HA combination treatment significantly improved arthralgia, reduced humoral and cellular immune responses and promoted angiogenesis, which led to an improvement in histological parameters, compared with PRP or HA injections alone. These results suggest that PRP and HA serve a critical therapeutic role in knee osteoarthritis progression and highlights their potential for the treatment of knee osteoarthritis in the future.

\section{Materials and methods}

Patients. Patients with knee osteoarthritis (age, 22-72 years; 170 females and 190 males) with a Karnofsky performance status of $\geq 80 \%$ (patients have difficulty walking by themselves and have knee pain) (22) were randomly divided into four groups and once-weekly, double-blind trials were conducted in Xi'an Jiangtong University College of Medicine (Xi'an, China). The inclusion/exclusion criteria, and allocation method are described in previously published studies $(23,24)$. Patients with knee osteoarthritis received PRP $(2,4,8,10,12$ and 14 ml, Sigma-Aldrich; Merck KGaA, Darmstadt, Germany), HA (0.10, 0.15, 0.20, 0.25 and 0.30 mg, Sigma-Aldrich; Merck $\mathrm{KGaA}$ ), combination treatment or placebo (normal saline, Sigma-Aldrich; Merck KGaA) through intralesional injections as referenced previously (23). The current phase-III study (XAJT006999781) was carried out in strict accordance with the recommendations in the Guide for Xi'an Jiangtong University College of Medicine between February 2009 and October 2014 (25). All patients were required to review trial protocols, amendments and provide informed consent. Ethical approval was granted by the Ethics Committee of Xi'an Jiangtong University College of Medicine (24).

Study design. The double-blind study was carried out in three phases: Baseline stage, the double-blind treatment phase (4-week dose-titration treatment, PRP, 2, 4, 8, 10, 12 and $14 \mathrm{ml}, \mathrm{HA}, 0.10,0.15,0.20,0.25$ and $0.30 \mathrm{mg}$ ) and 52-week post-treatment (PRP, 8ml, HA, $0.2 \mathrm{mg}$ ) for patients with knee osteoarthritis who volunteered to complete the ongoing extension study. Patients were randomly sorted into groups where they underwent once-weekly, double-blind treatment with HA $(n=88)$, PRP $(n=104)$, combination therapy of HA and PRP $(n=96)$ or a placebo $(n=72)$. Patients with knee osteoarthritis continued treatment with PRP $(8 \mathrm{ml})$, HA $(0.20 \mathrm{mg})$, combination (PRP: $8 \mathrm{ml}, \mathrm{HA}$ : $0.20 \mathrm{mg}$ ) or placebo throughout the maintenance period (52 weeks).

Outcomes measures. A Western Ontario and McMaster Universities Arthritis Index (WOMAC) questionnaire (26) for pain, two items for stiffness and 17 items for assessing functional limitation and the function of patients with knee osteoarthritis. The data was recorded and the degree of lesion was calculated.

ELISA. Plasma samples were prepared immediately using centrifugation at $2,000 \times \mathrm{g}$ at $4^{\circ} \mathrm{C}$ for $10 \mathrm{~min}$. Serum levels of TNF- $\alpha$ (cat no. MBS6080, Thermo Fisher Scientific, Inc., Waltham, MA, USA), IL-1 $\beta$ (cat no. MBS700340, Thermo Fisher Scientific, Inc.), IL-6 (cat no. MBS3205, Thermo Fisher Scientific,Inc.), IL-17A (cat no. DY-5194, Bio-Rad Laboratories, Inc., Hercules, CA, USA), RANKL (cat no. DY626, Bio-Rad Laboratories, Inc.), PD-ECGF (cat no. DY229-05, Bio-Rad), VEGF (cat no. DVE00, Bio-Rad Laboratories, Inc.) and IL-10 (cat no. MBS910284, Thermo Fisher Scientific, Inc.) were analyzed using ELISA kits according to the manufacturer's protocol. The serum concentration levels of these cytokines were measured using micro-plate reader at a wavelength of $570 \mathrm{~nm}$.

Efficacy and safety assessments. Efficacy assessments, including WOMAC scores or Karnofsky performance were analyzed in patients with knee osteoarthritis at baseline, during the 52-week and double-blind period in the PRP (8 ml), HA (0.20 mg), combination (PRP: $8 \mathrm{ml}$, HA: $0.20 \mathrm{mg}$ ) or placebo treatment groups. In addition, the overall safety and pharmacokinetic analysis were conducted according to previous clinical studies (25-27). Safety assessments of the most frequent treatment-emergent adverse events were evaluated in all randomized patients who received the study drug and had undergone at least one post-dose safety assessment. Dose-response analysis was conducted at the time of the last drug injection. Common Toxicity Criteria grades for hypertension and proteinuria were determined by the National Institute Common Terminology Criteria (28).

Statistical analysis. Data are expressed as the mean \pm standard deviation. Statistical analysis was performed using a Student's t-test for unpaired data. Comparisons of data between multiple groups were performed using one-way analysis of variance followed by a Dunnett's t test. Treatment effect was presented as median reduction in knee osteoarthritis over the treatment period. Robust nonparametric Responder rates and treatment-emergent adverse events were analyzed using a $\chi^{2}$ test. $\mathrm{P}<0.05$ was considered to indicate a statistically significant difference.

\section{Results}

Patient characteristics. There were 360 patients with knee osteoarthritis (mean age, 48 years) who were candidates for intra-articular injection in the present study. All patients were randomly divided into four groups and treated with HA $(n=88)$, 
Table I. Patient characteristics.

\begin{tabular}{lccrr}
\hline & \multicolumn{3}{c}{ Study groups } \\
\cline { 2 - 5 } Characteristic & PRP & HA & Combination & Placebo \\
\hline Number, n (\%) & $104(28.9)$ & $88(24.4)$ & $96(26.7)$ & $72(20.0)$ \\
Age, mean \pm SD & $46.2 \pm 8.6$ & $51.5 \pm 9.3$ & $46.5 \pm 7.5$ & $56.2 \pm 8.4$ \\
Gender (\%) & & & & $30(41.7)$ \\
Female, $\mathrm{n}(\%)$ & $54(51.9)$ & $40(45.5)$ & $46(47.9)$ & $42(58.3)$ \\
Male, $\mathrm{n}(\%)$ & $50(48.1)$ & $48(54.5)$ & $50(52.1)$ & \\
\hline
\end{tabular}

PRP, platelet-rich plasma; HA, hyaluronic acid; SD, standard deviation.

Table II. Treatment-emergent adverse events following platelet-rich plasma (PRP) treatment.

\begin{tabular}{lcccc}
\hline Adverse event & Total $(\mathrm{n}=28)$ & PRP $(2-4 \mathrm{ml} ; \mathrm{n}=8)$ & PRP $(8-12 \mathrm{ml} ; \mathrm{n}=12)$ & PRP $(14 \mathrm{ml} ; \mathrm{n}=8)$ \\
\hline Hypertension & 4 & 1 & 1 & 2 \\
Diarrhea & 3 & 1 & 1 & 1 \\
Proteinuria & 5 & 1 & 2 & 2 \\
Vomiting & 1 & 0 & 1 & 0 \\
Rash & 2 & 0 & 1 & 1 \\
Fatigue & 3 & 0 & 1 & 2 \\
Constipation & 4 & 1 & 1 & 1 \\
Hypertriglyceridemia & 3 & 1 & 1 & 1 \\
Edema peripheral & 3 & 1 & 1 & 2 \\
\hline
\end{tabular}

PRP ( $n=104)$, combination therapy of HA and PRP ( $n=96)$ or a placebo $(n=72)$. The numbers of male and female patients were approximately equal. The characteristics of patients with knee osteoarthritis are presented in Table I. Overall, 277 (75\%) patients with knee osteoarthritis completed the maintenance period of the phase III study, the other $25 \%$ stopped the study due to side effects.

Duration of treatment, dose-limiting toxicities and maximum tolerated dose. Median overall duration of PRP and HA treatments was 8 weeks. Patients underwent treatments with at least one of the following doses: 2, 4, 8, 10, 12 and $14 \mathrm{ml}$ for PRP; and $0.10,0.15,0.20,0.25$ and $0.30 \mathrm{mg}$ for HA. The data presented in Table II demonstrated that $12 \mathrm{ml}$ PRP once a week was identified as the maximum tolerated dose (MTD) and $16 \mathrm{ml}$ of PRP once a week was identified as dose-limiting toxicity (DLT) (29). Doses of 0.25 and $0.30 \mathrm{mg}$ HA were identified as the MTD and DLT, respectively (Table III). The common treatment-emergent adverse events of PRP or HA injection were hypertension, diarrhea, vomiting, rash, proteinuria, fatigue, constipation, hypertriglyceridemia and edema peripheral. A total of 60 patients with knee osteoarthritis required a reduction in drug dose for cumulative toxicity following treatment with MTD dose. Therefore, most patients were enrolled at a dose of $8 \mathrm{ml}$ PRP and $0.20 \mathrm{mg}$ HA for further examination of the tolerability and therapeutic effects of patients with knee osteoarthritis.
Treatment-emergent adverse events of PRP, HA and combination treatment. Patients with knee osteoarthritis who received at least one dose of study therapy with a post-baseline safety evaluation were included in the safety population. Following the last dose of PRP, it was observed that the most common treatment-emergent adverse events of PRP, HA and combination treatment (PRP, $8 \mathrm{ml}$; HA, $0.20 \mathrm{mg}$ ) were hypertension and proteinuria ( $\geq 10 \%$ each; Table IV). The data for the $12 \mathrm{ml}(\mathrm{n}=28)$ and $14 \mathrm{ml}(\mathrm{n}=18)$ PRP doses are not shown as there were more side effects, including hypertension, proteinuria, constipation and diarrhea and few patients were treated at these dose levels. Of the 360 patients enrolled in the current study, 118 patients with knee osteoarthritis completed the overall maintenance period of the phase III study.

Efficacy of combination PRP and HA treatment. The clinical outcomes of combination treatment of PRP and HA were analyzed. Preliminary clinical analyses indicated that pain was markedly improved in drug-treatment groups compared with the placebo group. Pain was reduced more in patients who received PRP treatment compared with patients who received HA treatment in the 52-week observation $(\mathrm{P}<0.01)$. Combination treatment of PRP and HA improved pain, physical function, stiffness and total WOMAC score compared with PRP treatment or HA treatment alone compared to the baseline (Table V). These clinical outcomes indicate that 
Table III. Treatment-emergent adverse events following hyaluronic acid (HA) treatment.

\begin{tabular}{lcccc}
\hline Adverse event & Total $(\mathrm{n}=30)$ & $\mathrm{HA}(0.10-0.15 \mathrm{mg} ; \mathrm{n}=8)$ & $\mathrm{HA}(0.20-0.25 \mathrm{mg} ; \mathrm{n}=15)$ & $\mathrm{HA}(0.30 \mathrm{mg} ; \mathrm{n}=7)$ \\
\hline Hypertension & 5 & 1 & 3 & 1 \\
Diarrhea & 2 & 0 & 1 & 1 \\
Proteinuria & 4 & 1 & 1 & 1 \\
Vomiting & 2 & 0 & 1 & 1 \\
Rash & 2 & 0 & 1 & 1 \\
Fatigue & 2 & 0 & 1 & 1 \\
Constipation & 3 & 1 & 1 & 1 \\
Hypertriglyceridemia & 3 & 0 & 1 & 1 \\
Edema peripheral & 2 & 0 & & 1 \\
\hline
\end{tabular}

Table IV. Common Toxicity Criteria grades for hypertension and proteinuria.

\begin{tabular}{lcccc}
\hline Adverse event & Total $(\mathrm{n}=72)$ & PRP $(\mathrm{n}=28)$ & HA $(\mathrm{n}=30)$ & Combination $(\mathrm{n}=14)$ \\
\hline Hypertension & 11 & 4 & $5^{\mathrm{a}}$ & 2 \\
Grade 1 & 5 & 2 & 2 & 1 \\
Grade 2 & 4 & 1 & 1 & 0 \\
Grade 3 & 2 & 1 & $4^{\mathrm{b}}$ & 2 \\
Proteinuria & 11 & $5^{\mathrm{b}}$ & 2 & 1 \\
Grade 1 & 5 & 2 & 1 & 1 \\
Grade 2 & 3 & 1 & 1 & 0 \\
Grade 3 & 3 & 2 & & \\
\hline
\end{tabular}

Treatment-emergent adverse events were analyzed by $\chi^{2}$ test. ${ }^{a} \mathrm{P}<0.05$, HA vs. Combination, ${ }^{\mathrm{b}} \mathrm{P}<0.05$, PRP or HA vs. Combination. HA, hyaluronic acid; PRP, platelet-rich plasma.

Table V. WOMAC scores during the study period.

WOMAC score, mean (SD)

\begin{tabular}{|c|c|c|c|c|}
\hline \multirow[b]{2}{*}{ Group } & \\
\hline & Pain & Stiffness & Physical function & Total \\
\hline \multicolumn{5}{|l|}{ PRP } \\
\hline Baseline & $8.86(3.14)$ & $2.67(1.76)$ & $24.68(12.63)$ & $36.21(17.53)$ \\
\hline Week 52 & $4.15(3.08)$ & $1.44(0.84)$ & $14.66(11.28)$ & $20.25(15.20)$ \\
\hline Changes & $4.67(3.07)^{\mathrm{g}}$ & $1.19(1.26)^{\mathrm{g}}$ & $9.98(11.92)^{\mathrm{g}}$ & $15.84(16.59)^{\mathrm{h}}$ \\
\hline P-value & $<0.0001^{\mathrm{a}}$ & $<0.0001^{\mathrm{a}}$ & $<0.0001^{\mathrm{a}}$ & $<0.0001^{\mathrm{a}}$ \\
\hline \multicolumn{5}{|l|}{ HA } \\
\hline Baseline & $8.91(3.82)$ & $2.78(1.58)$ & $25.72(12.32)$ & $37.41(16.72)$ \\
\hline Week 52 & $5.26(3.75)$ & $2.25(1.38)$ & $18.89(12.85)$ & $26.40(16.98)$ \\
\hline Changes & $3.61(3.75)$ & $0.49(1.44)$ & $6.79(12.55)$ & $10.89(17.74)$ \\
\hline P-value & 0.01 & 0.01 & 0.01 & 0.01 \\
\hline \multicolumn{5}{|c|}{ Combination } \\
\hline Baseline & $8.94(5.12)$ & $2.81(1.47)$ & $26.46(10.66)$ & $38.21(17.25)$ \\
\hline Week 52 & $3.32(2.44)$ & $0.85(0.68)$ & $10.23(8.61)$ & $14.40(11.73)$ \\
\hline Changes & $5.58(2.64)^{\mathrm{c}, \mathrm{e}}$ & $1.92(1.04)^{\mathrm{f}}$ & $16.19(9.60)^{\mathrm{d}, \mathrm{f}}$ & $23.69(13.28)^{\mathrm{d}, \mathrm{f}}$ \\
\hline P-value & $<0.0001^{\mathrm{b}}$ & $<0.0001^{\mathrm{b}}$ & $<0.0001^{\mathrm{b}}$ & $<0.0001^{\mathrm{b}}$ \\
\hline
\end{tabular}

HA, hyaluronic acid; PRP, platelet-rich plasma; WOMAC, Western Ontario and McMaster Universities Arthritis Index; SD, standard deviation. ${ }^{\mathrm{a}} \mathrm{P}<0.0001$, Week 52 vs. baseline in PRP group. ${ }^{\mathrm{b}} \mathrm{P}<0.0001$, Week 52 vs. baseline in the combination group. ${ }^{\mathrm{c}} \mathrm{P}<0.05,{ }^{\mathrm{d}} \mathrm{P}<0.01$, Combination vs. PRP; ${ }^{\mathrm{P}}<0.05,{ }^{\mathrm{f}} \mathrm{P}<0.01$, Combination vs. HA; ${ }^{\mathrm{g}}<0.05,{ }^{\mathrm{h}} \mathrm{P}<0.01, \mathrm{PRP}$ vs. HA. 


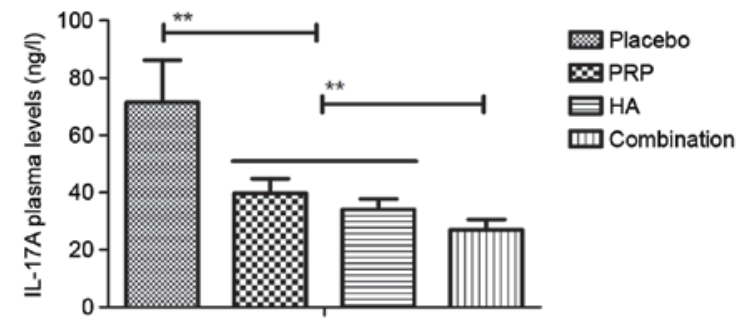

Figure 1. Effect of combination of PRP and HA treatment on plasma concentration levels of IL-17A in patients with knee osteoarthritis. Data are presented as the mean \pm standard deviation. ${ }^{* *} \mathrm{P}<0.01$. IL-17A, interleukin-17A; PRP, platelet-rich plasma; HA, hyaluronic acid.

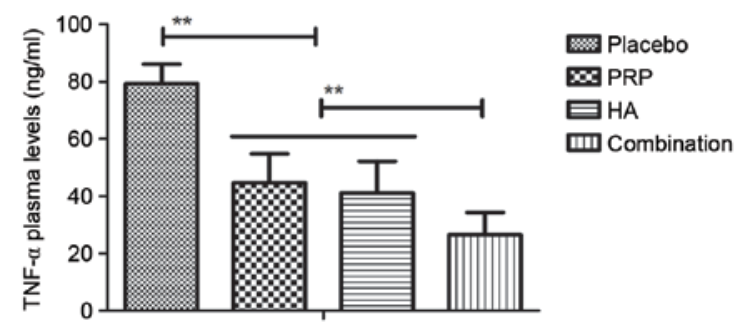

Figure 2. Effect of combination of PRP and HA treatment on plasma concentration levels of TNF- $\alpha$ in patients with knee osteoarthritis. Data are presented as the mean \pm standard deviation. ${ }^{* *} \mathrm{P}<0.01$. TNF- $\alpha$, tumor necrosis factor- $\alpha$; PRP, platelet-rich plasma; HA, hyaluronic acid.

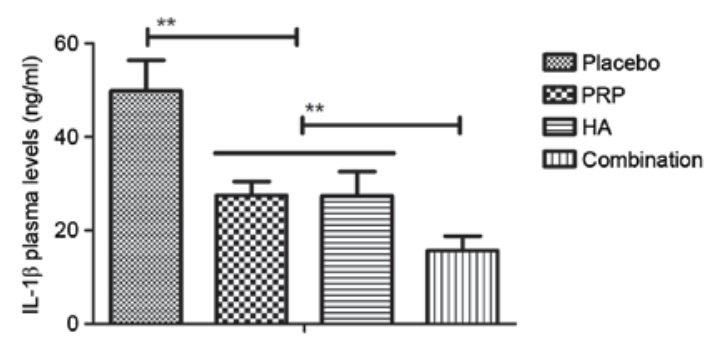

Figure 3. Effect of combination of PRP and HA treatment on plasma concentration levels of IL-1 $\beta$ in patients with knee osteoarthritis. Data are presented as the mean \pm standard deviation. ${ }^{* *} \mathrm{P}<0.01$. IL-1 $\beta$, interleukin- $1 \beta$; PRP, platelet-rich plasma; HA, hyaluronic acid.

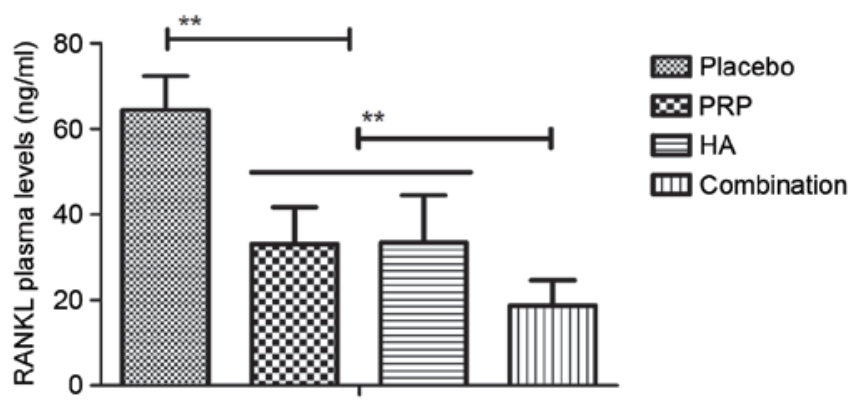

Figure 4. Effect of combination of PRP and HA treatment on plasma concentration levels of RANKL in patients with knee osteoarthritis. Data are presented as the mean \pm standard deviation. ${ }^{* *} \mathrm{P}<0.01$. RANKL, receptor activator of nuclear factor- $\mathrm{kB}$ ligand; PRP, platelet-rich plasma; HA, hyaluronic acid.

PRP ( $8 \mathrm{ml})$ and HA (0.20 mg) combination therapy improves the clinical features of knee osteoarthritis.

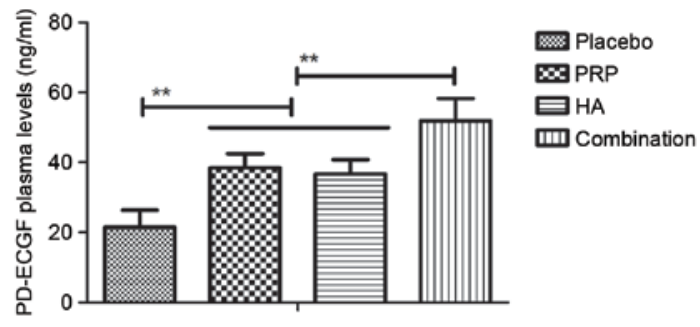

Figure 5. Effect of combination PRP and HA treatment on plasma concentration levels of PD-ECGF in patients with knee osteoarthritis. Data are presented as the mean \pm standard deviation. ${ }^{* *} \mathrm{P}<0.01$. ECGF, platelet derived-endothelial cell growth factor; PRP, platelet-rich plasma; HA, hyaluronic acid.

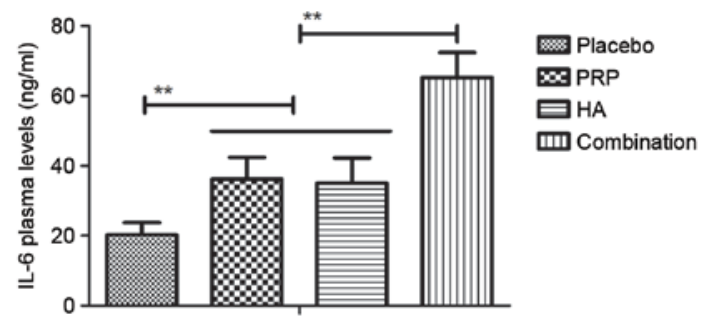

Figure 6. Effect of combination PRP and HA treatment on plasma concentration levels of IL-6 in patients with knee osteoarthritis. Data are presented as the mean \pm standard deviation. ${ }^{* *} \mathrm{P}<0.01$. IL-6, interleukin-6; PRP, platelet-rich plasma; HA, hyaluronic acid.

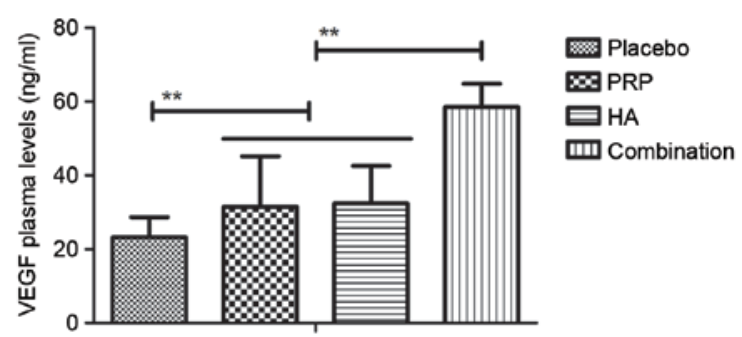

Figure 7. Effect of combination PRP and HA treatment on plasma concentration levels of VEGF in patients with knee osteoarthritis. Data are presented as the mean \pm standard deviation. ${ }^{* *} \mathrm{P}<0.01$. VEGF, vascular endothelial growth factor; PRP, platelet-rich plasma; HA, hyaluronic acid.

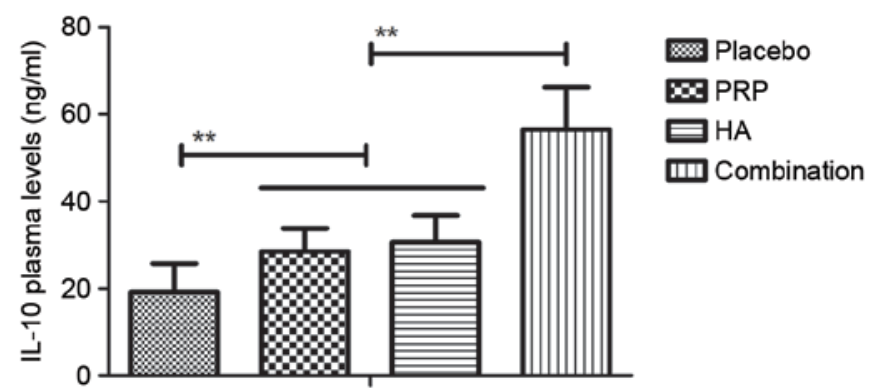

Figure 8. Effect of combination PRP and HA treatment on plasma concentration levels of IL-10 in patients with knee osteoarthritis. Data are presented as the mean \pm standard deviation. ${ }^{* *} \mathrm{P}<0.01$. IL-10, interleukin-10; PRP, platelet-rich plasma; HA, hyaluronic acid.

Anti-inflammation effects of combination PRPandHAtreatment for patients with knee osteoarthritis. The level of serum inflammatory cytokines in patients with knee osteoarthritis following 
PRP and/or HA treatments was then measured. Plasma concentration levels of interleukin (IL)-17A, tumor necrosis factor- $\alpha$, IL-1 $\beta$ and receptor activator of nuclear factor $\kappa-B$ ligand were downregulated in patients with knee osteoarthritis following treatment with PRP or HA and further downregulated following combination treatment with PRP and HA (all P<0.01; Figs. 1-4). The plasma concentration of platelet derived-endothelial cell growth factor, IL-6, vascular endothelial growth factor and IL-10 were upregulated in patients with knee osteoarthritis following treatment with PRP or HA and were further upregulated following combination treatment with PRP and HA (all $\mathrm{P}<0.01$; Figs. 5-8). These results suggest that combination PRP and HA treatment inhibits inflammation for patients with knee osteoarthritis.

\section{Discussion}

Reports have indicated that inflammatory cytokines serve a critical function in the induction and development of osteoarthritis $(30,31)$. PRP is an autologous and multifunctional platelet concentrate of the blood that contains highly concentrated platelets and high levels of cell growth factors. PRP promotes synovial cell proliferation and differentiation, and promotes recovery of cartilage morphology. Clinically, Battaglia et al (32) has demonstrated the efficacy of ultrasound-guided intra-articular injections of PRP vs. HA for hip osteoarthritis. Laudy et al (33) has suggested that PRP injections are beneficial to patients with knee osteoarthritus based on a reduction in pain, improvement in function, global assessment and changes regarding joint imaging. Meheux et al (34) demonstrated that treatment with PRP injection significantly improved validated patient-reported outcomes in patients with symptomatic knee osteoarthritis at 6 and 12 months post-injection, and indicated similarities and differences in outcomes based on the PRP formulations used in the analyzed studies. These clinical reports suggest that PRP exhibited a potential efficacy for treating osteoarthritis.

HA is responsible for the viscoelastic and lubricant capabilities of the synovial fluid in joints. It serves a key function in metabolism in the joints and mechanical support, which stimulates chondrocyte metabolism and cartilage matrix components synthesis as well as inflammatory processes (35). Clinical research has indicated that intra-articular HA injections in patients with knee osteoarthritis are associated with pain relief, quality of life, survival time, clinical effect and a longer period of time prior to the onset of knee arthroplasty (16). In addition, the efficacy and safety of HA in the management of osteoarthritis has been investigated using real-life setting trials and surveys (17). However, single intra-articular HA injections did not achieve the ideal therapeutic effect for patients with osteoarthritis.

Previous studies have compared the clinical efficacy of PRP vs. HA for treatment of knee osteoarthritis and determined that PRP presented more notable improvements in physical function, stiffness and total WOMAC $(14,36)$. Coincidentally, Kon et al (37) investigated PRP intra-articular injection vs. HA viscosupplementation in treatments for cartilage pathology from early degeneration to osteoarthritis, and outcomes suggested that PRP was superior to HA treatment for patients with cartilage pathology. However, the efficacy and safety of combination treatment with PRP and HA for patients with knee osteoarthritis remains unknown.

In the present study, the clinical efficacy of combination treatment with PRP and HA for patients with knee osteoarthritis was investigated in a phase-III clinical study. Following an 8-week baseline period, patients with knee osteoarthritis were randomized into groups undergoing once-weekly, double-blind treatment with PRP, HA, combined therapy or a placebo. Although previous studies indicated that patients with knee osteoarthritis treated with PRP or HA exhibited regulated plasma concentrations of inflammatory factors and pro-angiogenic factors, the clinical outcomes of combined PRP and HA have not been investigated $(27,38)$. The current study was performed to evaluate the clinical application of combination treatment with PRP and HA. Responses to treatment were assessed by median percent reduction in arthralgia, which was improved with PRP and/or HA treatments compared with the placebo group. Hypertension and proteinuria were the treatment-emergent adverse events with the highest incidence following treatment with PRP or HA alone. The results demonstrated that PRP and/or HA alleviated knee osteoarthritis and reduced the humoral and cellular immune responses, which subsequently led to beneficial effects on histological parameters. The clinical outcomes revealed a significant improvement in all the variables of WOMAC following combination treatment with PRP and HA.

In conclusion, although the direct effects of different drugs on knee osteoarthritis have been demonstrated previously, it is critical that the overall role of PRP and HA in affecting entire joint cytokines homeostasis is investigated (39). Clinical outcomes of the current study demonstrated that PRP and HA are potential novel therapeutic options for treating knee osteoarthritis and an increasing number of clinical reports continue to indicate promising results. Of note, the results of the current study suggest that pharmacokinetic interactions of PRP and $\mathrm{HA}$ are important determinants in optimizing therapies for treating knee osteoarthritis. Therefore, clinicians are required to monitor clinical responses and tolerability when patients are treated with PRP and HA. The results indicate that patients with knee osteoarthritis treated with PRP and HA exhibited beneficial effects on body pain, and alleviated arthralgia, cartilage destruction and bone damage.

\section{Competing interests}

The authors declare that they have no competing interests.

\section{References}

1. Onuora S: Osteoarthritis: Molecular imaging detects activated macrophages. Nat Rev Rheumatol 12: 313, 2016.

2. Ma YW, Jiang DL, Zhang D, Wang XB and Yu XT: Radial extracorporeal shock wave therapy in a person with advanced osteonecrosis of the femoral head. Am J Phys Med Rehabil 95: e133-e139, 2016.

3. Lee GW, Park KS, Kim DY, Lee YM, Eshnazarov KE and Yoon TR: Results of total hip arthroplasty after core decompression with tantalum rod for osteonecrosis of the femoral head. Clin Orthop Surg 8: 38-44, 2016.

4. Mutlu H, Karaca H, Akca Z and Torun YA: Should fish test be performed to all patients with breast cancer? Med Sci (Turkey) 2: 539-547, 2013. 
5. Roemer FW, Kwoh CK, Hannon MJ, Hunter DJ, Eckstein F, Grago J, Boudreau RM, Englund M and Guermazi A: Partial meniscectomy is associated with increased risk of incident radiographic osteoarthritis and worsening cartilage damage in the following year. Eur Radiol 27: 404-413, 2017.

6. Tang H, He S, Zhang X, Luo S, Zhang B, Duan X, Zhang Z, Wang W, Wang Y and Sun Y: A network pharmacology approach to uncover the pharmacological mechanism of XuanHuSuo powder on osteoarthritis. Evid Based Complement Alternat Med 2016: 3246946, 2016.

7. Poquet N, Williams $\mathrm{M}$ and Bennell KL: Exercise for osteoarthritis of the hip. Phys Ther 96: 1689-1694, 2016.

8. Maricar N, Callaghan MJ, Parkes MJ, Felson DT and O'Neill TW: Clinical assessment of effusion in knee osteoarthritis-A systematic review. Semin Arthritis Rheum 45: 556-563, 2016.

9. Beumer L, Wong J, Warden SJ, Kemp JL, Foster P and Crossley KM: Effects of exercise and manual therapy on pain associated with hip osteoarthritis: A systematic review and meta-analysis. Br J Sports Med 50: 458-463, 2016

10. Smyth NA, Haleem AM, Ross KA, Hannon CP, Murawski CD Do HT and Kennedy JG: Platelet-rich plasma may improve osteochondral donor site healing in a rabbit model. Cartilage 7: 104-111, 2016.

11. Bembo F, Eraud J, Philandrianos C, Bertrand B, Silvestre A, Veran J, Sabatier F, Magalon G and Magalon J: Combined use of platelet rich plasma \& micro-fat in sport and race horses with degenerative joint disease: Preliminary clinical study in eight horses. Muscles Ligaments Tendons J 6: 198-204, 2016.

12. Fu CJ, Sun JB, Bi ZG, Wang XM and Yang CL: Evaluation of platelet-rich plasma and fibrin matrix to assist in healing and repair of rotator cuff injuries: A systematic review and meta-analysis. Clin Rehabil 31: 158-172, 2017.

13. Vannini F, Di Matteo B and Filardo G: Platelet-rich plasma to treat ankle cartilage pathology-from translational potential to clinical evidence: A systematic review. J Expe Orthop 2: 2,2015 .

14. Sadabad HN, Behzadifar M, Arasteh F, Behzadifar M and Dehghan HR: Efficacy of platelet-rich plasma versus hyaluronic acid for treatment of knee osteoarthritis: A systematic review and meta-analysis. Electron Physician 8: 2115-2122, 2016.

15. Khoshbin A, Leroux T, Wasserstein D, Marks P, Theodoropoulos J, Ogilvie-Harris D, Gandhi R, Takhar K, Lum G and Chahal J: The efficacy of platelet-rich plasma in the treatment of symptomatic knee osteoarthritis: A systematic review with quantitative synthesis. Arthroscopy 29: 2037-2048, 2013.

16. Ong KL, Anderson AF, Niazi F, Fierlinger AL, Kurtz SM and Altman RD: Hyaluronic acid injections in medicare knee osteoarthritis patients are associated with longer time to knee arthroplasty. J Arthroplasty 31: 1667-1673, 2016.

17. Maheu E, Rannou F and Reginster JY: Efficacy and safety of hyaluronic acid in the management of osteoarthritis: Evidence from real-life setting trials and surveys. Semin Arthritis Rheum 45 (4 Suppl): S28-S33, 2016.

18. Altman R, Lim S, Steen RG and Dasa V: Correction: Hyaluronic acid injections are associated with delay of total knee replacement surgery in patients with knee osteoarthritis: Evidence from a large U.S. health claims database. PLoS One 11: e0148591, 2016.

19. Witteveen AG, Hofstad CJ and Kerkhoffs GM: Hyaluronic acid and other conservative treatment options for osteoarthritis of the ankle. Cochrane Database Syst Rev 10: CD010643, 2015.

20. Panuccio E, Memeo A and Richetta S: Evaluation of the combined treatment of oral viscosupplementation with hyaluronic acid intra-articular injection on symptomatic knee osteoarthritis. Clin Ter 166: e321-e326, 2015 (In Italian).

21. Lubowitz JH: Editorial commentary: Knee hyaluronic acid viscosupplementation reduces osteoarthritis pain. Arthroscopy 31 $2046,2015$.

22. Okita Y, Narita Y, Miyahara R, Miyakita Y, Ohno M and Shibui S: Health-related quality of life in long-term survivors with grade II gliomas: The contribution of disease recurrence and Karnofsky performance status. Jpn J Clin Oncol 45: 906-913, 2015 .
23. Raeissadat SA, Rayegani SM, Hassanabadi H, Fathi M, Ghorbani E, Babaee M and Azma K: Knee osteoarthritis injection choices: Platelet-rich plasma (PRP) versus hyaluronic acid (A one-year randomized clinical trial). Clin Med Insights Arthritis Musculoskeletal Disord 8: 1-8, 2015.

24. Rodriguez-Merchan EC: Intraarticular injections of platelet-rich plasma (PRP) in the management of knee osteoarthritis. Arch Bone Jt Surg 1: 5-8, 2013

25. Filardo G, Kon E, DI Matteo B, DI Marino A, Sessa A, Merli ML and Marcacci M: Leukocyte-poor PRP application for the treatment of knee osteoarthritis. Joints 1: 112-120, 2014.

26. Hmamouchi I, Allali F, Tahiri L, Khazzani H, Mansouri LE, Ali Ou Alla S, Abouqal R and Hajjaj-Hassouni N: Clinically important improvement in the WOMAC and predictor factors for response to non-specific non-steroidal anti-inflammatory drugs in osteoarthritic patients: A prospective study. BMC Res Notes 5: $58,2012$.

27. Gobbi A, Lad D and Karnatzikos G: The effects of repeated intra-articular PRP injections on clinical outcomes of early osteoarthritis of the knee. Knee Surg Sports Traumatol Arthrosc 23: 2170-2177, 2015

28. Trotti A, Colevas AD, Setser A, Rusch V, Jaques D, Budach V, Langer C, Murphy B, Cumberlin R, Coleman CN and Rubin P: CTCAE v3.0: Development of a comprehensive grading system for the adverse effects of cancer treatment. Semin Radiat Oncol 13: 176-181, 2003.

29. Nations KR, Bursi R, Dogterom P, Ereshefsky L, Gertsik L, Mant T and Schipper J: Maximum tolerated dose evaluation of the AMPA modulator Org 26576 in healthy volunteers and depressed patients: A summary and method analysis of bridging research in support of phase II dose selection. Drugs R D 12: 127-139, 2012.

30. Hodge JA, Kawabata TT, Krishnaswami S, Clark JD, Telliez JB, Dowty ME, Menon S, Lamba M and Zwillich S: The mechanism of action of tofacitinib-an oral Janus kinase inhibitor for the treatment of rheumatoid arthritis. Clin Exp Rheumatol 34: 318-328, 2016.

31. van der Goes MC, Jacobs JW and Bijlsma JW: Rediscovering the therapeutic use of glucocorticoids in rheumatoid arthritis. Curr Opin Rheumatol 28: 289-296, 2016.

32. Battaglia M, Guaraldi F, Vannini F, Rossi G, Timoncini A, Buda R and Giannini S: Efficacy of ultrasound-guided intra-articular injections of platelet-rich plasma versus hyaluronic acid for hip osteoarthritis. Orthopedics 36: e1501-e1508, 2013.

33. Laudy AB, Bakker EW, Rekers M and Moen MH: Efficacy of platelet-rich plasma injections in osteoarthritis of the knee: A systematic review and meta-analysis. Br J Sports Med 49: 657-672, 2015

34. Meheux CJ, McCulloch PC, Lintner DM, Varner KE and Harris JD: Efficacy of intra-articular platelet-rich plasma injections in knee osteoarthritis: A systematic review. Arthroscopy 32: 495-505, 2016.

35. Altman RD, Manjoo A, Fierlinger A, Niazi F and Nicholls M: The mechanism of action for hyaluronic acid treatment in the osteoarthritic knee: A systematic review. BMC Musculoskeletal Disord 16: 321, 2015.

36. Ahadi T and Abtahi M: Platelet-rich plasma versus hyaluronic acid. Arthroscopy 28: 1585; author reply 1585-1586, 2012.

37. Kon E, Mandelbaum B, Buda R, Filardo G, Delcogliano M, Timoncini A, Fornasari PM, Giannini S and Marcacci M: Platelet-rich plasma intra-articular injection versus hyaluronic acid viscosupplementation as treatments for cartilage pathology: From early degeneration to osteoarthritis. Arthroscopy 27: 1490-1501, 2011.

38. Lùrati A, Laria $\mathrm{A}$, Mazzocchi $\mathrm{D}$, Re KA, Marrazza $\mathrm{M}$ and Scarpellini M: Effects of hyaluronic acid (HA) viscosupplementation on peripheral Th cells in knee and hip osteoarthritis. Osteoarthritis Cartilage 23: 88-93, 2015.

39. Görmeli G, Görmeli CA, Ataoglu B, Çolak C, Aslantürk O and Ertem K: Multiple PRP injections are more effective than single injections and hyaluronic acid in knees with early osteoarthritis: A randomized, double-blind, placebo-controlled trial. Knee Surg Sports Traumatol Arthrosc 25: 958-965, 2017. 